\title{
Spiral computed tomography of pulmonary embolism
}

\author{
C.J. Herold
}

\begin{abstract}
Spiral computed tomography of pulmonary embolism. C.J. Herold. (C)ERS Journals Ltd 2002.

ABSTRACT: Within the last several years, spiral computed tomography angiography (SCTA) of the pulmonary arteries has emerged as a noninvasive angiographic modality for the evaluation of patients with suspected pulmonary embolism (PE). SCTA is based on continuous computed tomography (CT) data acquisition during patient transport through the rotating $\mathrm{X}$-ray tube and detector system, where scanning is performed in the time period in which the injected contrast material passes through the pulmonary arteries. Single detector spiral CT has a sensitivity of $\sim \mathbf{8 5}-\mathbf{9 0} \%$ and a specificity between $88-95 \%$. Sensitivity and specificity are very likely to increase with the use of multidetector spiral CT scanners that allow scanning of large lung volumes with a scan collimation as narrow as $1 \mathrm{~mm}$.

Currently, SCTA is most commonly used as a primary imaging method in patients with suspected $\mathrm{PE}$, and as a second-line method in cases with inconclusive ventilation/ perfusion scintigraphy results. SCTA has proven to be cost-effective, especially in combination with ultrasound of the lower extremities.

Limitations of the method include a decreased sensitivity for the detection of small isolated clots in the peripheral pulmonary arterial bed, and a potentially reduced image quality in patients with coexistent cardiopulmonary disorders.

Despite these limitations, several studies have now documented that, in patients with suspected pulmonary embolism, it is safe to withhold anticoagulation therapy if a spiral computed tomography exam of the pulmonary arteries is negative and no lower extremity venous thrombosis is present. In the future, multislice computed tomography scanning of the pulmonary arteries with multiplanar reformation and one-stop shopping, i.e. scanning of the pulmonary arteries and the lower extremity veins in a single session, will further enhance the role of computed tomography angiography in the examination of patients with suspected pulmonary embolism.

Eur Respir J 2002; 19: Suppl. 35, 13s-21s.
\end{abstract} \author{
Vienna, Vienna, Austria. \\ Correspondence: C.J. Herold \\ Dept of Radiology \\ University of Vienna \\ Waehringer Guertel 18-20 \\ A-1090 Vienna \\ Austria \\ Fax: 431404004894 \\ E-mail: christian.herold@ \\ akh-wien.ac.at
}

Dept of Radiology, University of

Keywords: Computed tomography computed tomography angiography pulmonary embolism

venous thromboembolism

Received: October 222001

Accepted October 232001
Pulmonary embolism (PE) is often encountered both in the hospital setting and in private clinical practice. Because untreated PE is associated with a greatly increased mortality risk, accurate diagnosis of $\mathrm{PE}$ is of critical importance $[1,2]$. However, because clinical findings, laboratory tests, and signs and symptoms at physical examination are often nonspecific, imaging methods are a significant part of the diagnostic evaluation of patients with suspected PE.

Since PE is not an isolated disorder but, rather, a symptom of the entire venous thromboembolism complex, imaging studies of the lower extremities and the pelvis, the most common source of pulmonary emboli, are a fundamental requirement for the diagnostic work-up of patients with suspected PE. Various imaging modalities have been attempted in this evaluation process within the last few decades. For many years, ventilation/perfusion $(V / Q)$ scintigraphy has served as the noninvasive procedure of choice for the assessment of patients with suspected PE. This is because a normal or near normal $V I Q$ scan rather reliably excludes embolic disease [3], while a high probability/abnormal result usually indicates $\mathrm{PE}$ in the context of corresponding clinical symptoms $[4,5]$. An intermediate or low probability (inconclusive) result at $V I Q$ scanning requires further imaging work-up, because the percentage of positive pulmonary angiograms has been shown to be as high as 33\% for intermediate probability results, and $16 \%$ for low probability results, respectively [5].

Pulmonary angiography has been used as the gold standard for the diagnosis of PE [4]. However, despite excellent sensitivity and specificity, the method has not gained widespread acceptance because it is invasive and clinicians are unwilling to expose patients, who often have unstable cardiovascular conditions, to a potentially risky procedure. This explains the results of a study in a large academic hospital which revealed that $92 \%$ of patients with low probability $V / Q$ scans and $70 \%$ of patients with intermediate probability scans were not further investigated, either with pulmonary angiography or with any other imaging modality [6]. Nevertheless, as this study showed, a large number of patients were treated on the basis of the best clinical guess method.

Within the last $10 \mathrm{yrs}$, technical advances in computed tomography (CT) have established the basis for the use of CT in the diagnosis of PE. Electron beam 
computed tomography and single slice and multislice spiral CT angiography (SCTA) have revolutionized the approach to the assessment of patients with suspected PE. With spiral CT, imaging acquisition times and total scan times are dramatically reduced compared to conventional $\mathrm{CT}$, and the pulmonary vascular tree can be investigated in a single breathhold scan at peak contrast opacification. Thus, modern $\mathrm{CT}$ imaging techniques provide direct visualization of pulmonary emboli within the pulmonary arteries [7, 8]. Since spiral CT is becoming more readily available, this article will focus on SCTA and discuss imaging technique, image interpretation, and the role of SCTA in the diagnosis of PE.

\section{Spiral computed tomography imaging technique}

The choice of the optimal imaging approach for the CT diagnosis of PE depends largely on the equipment at hand, specifically on whether single or multislice CT scanners are available. For each of these scanner systems, imaging protocols for the assessment of the pulmonary vasculature exist. In many institutions, single-slice SCTA is performed as a single contrast series through the thorax, if possible, using the single breath-hold technique [9], 90\% of patients evaluated for suspected PE can successfully suspend respiration for $25-30 \mathrm{~s}$, allowing for a single breath-hold scan. The $\sim 10 \%$ of patients who are unable to hold their breath, are evaluated during shallow breathing. In multislice CT scanning, data acquisition may take $\leqslant 20 \mathrm{~s}$ using a scan protocol that uses the narrowest possible collimation to provide the most detailed display of the pulmonary arteries. In dyspnoeic patients, multislice scanning can be shortened to $\sim 10 \mathrm{~s}$, based on a fast data acquisition protocol.

It is important to emphasize that the scan range, i.e. the lung volume scanned, should include the lung sections in which the subsegmental vessels are located. Practically, this means that CT scanning should cover a range between the costophrenic angles and the top of the aortic arch. Most institutions now prefer a caudocranial scanning direction rather than a craniocaudal data acquisition, because breathing artifacts are significantly less intensive in the upper portions of the lung (compared to the lower portions) when the patient breathes during the final phase of data acquisition. Breathing artifacts can also be reduced by shortening data acquisition through an increase in collimation and table feed.

In single-slice CT, imaging is performed most often using $120 \mathrm{kV}, 210-250 \mathrm{mAs}$, a slice thickness of $3 \mathrm{~mm}$, a table speed of $5 \mathrm{~mm} \cdot \mathrm{s}^{-1}$, (pitch 1.7), and a reconstruction index of $2 \mathrm{~mm}$. The advantage of increased pitch is that larger volumes can be scanned without loss of resolution [10]. Recently, the value of narrowing the scan collimation to $2 \mathrm{~mm}$ for the analysis of subsegmental vessels has been reported [11]. Such narrow collimation, however, may be more suited to a tailored approach using a predefined volume rather than for scanning the entire thorax. In multislice CT angiography, slice collimation can be significantly reduced in patients who are able to hold their breath. In these patients, scan protocols now involve a slice collimation of $1 \mathrm{~mm}$, a table increment of $7 \mathrm{~mm} \cdot$ rotation $^{-1}$, and a reconstruction index of $1 \mathrm{~mm}$. In dyspnoeic patients, slice collimation is set at $2.5 \mathrm{~mm}$, table increment at $15 \mathrm{~mm} \cdot$ rotation $^{-1}$, and reconstruction at $1.5 \mathrm{~mm}$. Data acquisition takes $15 \mathrm{~s}$ for a $20-\mathrm{cm}$ scan length with the "high detail" protocol, and $7 \mathrm{~s}$ for a $20-\mathrm{cm}$ scan length with the "high speed" protocol.

The time interval between the application of contrast material and the start of data acquisition, the scan delay, should be chosen according to the patient's clinical status. In patients who have no history, signs or symptoms of pulmonary arterial hypertension, right ventricular failure, or overall cardiac failure, a scan delay of $15 \mathrm{~s}$ is sufficient to allow for optimum vessel opacification. In patients with signs or symptoms of any of the aforementioned disorders, a scan delay between 15-30 s may be necessary and should be determined by a test bolus $(15 \mathrm{~mL})$ or the Smartprep technique, i.e. an automatically triggered contrast media injection.

Contrast material should be administered using a power injector. In most institutions, nonionic contrast media are preferred, particularly when the high flow technique is used. Although no optimum protocol has been defined, there are two basic approaches to contrast media injection in single-slice CT: the low concentration/high flow approach, in which contrast media with $150-200 \mathrm{mg}$ iodine $\cdot \mathrm{mL}^{-1}$ is injected with a rate of $4-5 \mathrm{~mL} \cdot \mathrm{s}^{-1}$; and the high concentration/low flow technique, in which contrast material with $300-$ $320 \mathrm{mg}$ iodine $\cdot \mathrm{mL}^{-1}$ is applied within $2-3 \mathrm{~mL} \cdot \mathrm{s}^{-1}$. Good to excellent results have been reported for both approaches $[7,9,12,13]$. The low concentration approach, however, has successfully reduced streak artifacts, which result from the inflowing contrast material in the superior vena cava and potentially limit the diagnostic accuracy in the pulmonary trunk and right pulmonary artery. Recently, some institutions, including the author's, have adopted a high concentration/high flow approach, and the author's experience with this technique is excellent (fig. 1). In multislice CT angiography, the volume of the injected contrast material can be reduced to $80-90 \mathrm{~mL}$ because of the shorter scanning times. Equally, the scan delay may be increased to $20 \mathrm{~s}$ to ensure that the entire pulmonary vascular bed is homogeneously opacified.

\section{Image interpretation}

Image interpretation is typically performed using both soft tissue (mediastinum) and pulmonary parenchymal window settings. This side-by-side analysis of images may help to differentiate pulmonary arteries that accompany the bronchi from venous structures, which, in the early phase of scanning, may be unenhanced [7]. In addition, cine-mode viewing provides a dynamic impression of the pulmonary arteries and is generally considered helpful in the evaluation of acute PE. The use of two-dimensional multiplanar reformations may also aid in the diagnosis [14, 15].

Pulmonary emboli within the pulmonary arteries 


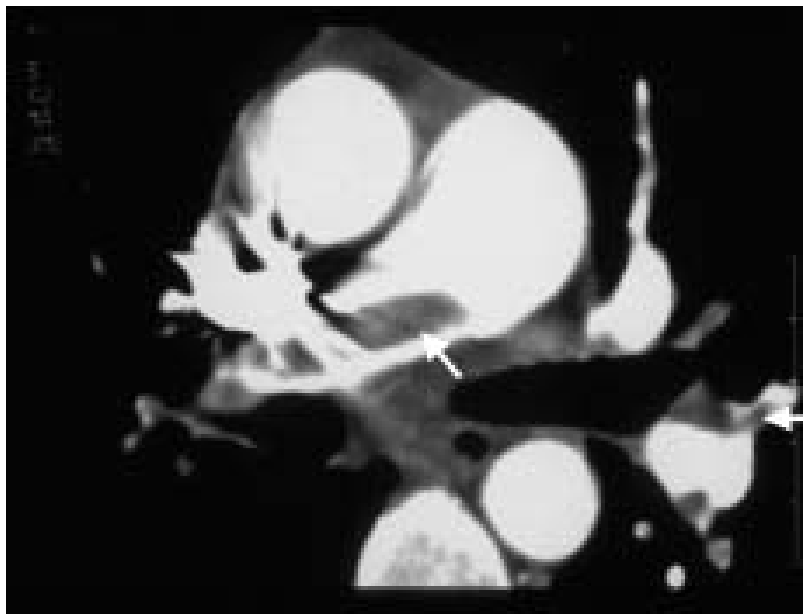

Fig. 1.- Spiral computed tomography (CT) angiogram in pulmonary embolism. Spiral CT angiogram in a 43-yr-old female, obtained with highly concentrated contrast material and high flow technique shows multiple pulmonary emboli (arrows) in the right and left pulmonary arterial system.

can be directly visualized with SCTA. Acute pulmonary emboli can be seen as hypoattenuating filling defects within the vessel, partly or completely surrounded by opacified blood, or as a complete filling defect that leaves the respective vessel totally unopacified [7] (fig. 1). Spiral CT can also help detect indirect signs of PE such as pleural-based densities, linear densities, or plate-like atelectases, central or peripheral dilatation of pulmonary arteries, and pleural effusions [16] (fig. 2). Seen as a whole, these ancillary parenchymal findings are detected in the majority of patients with PE, but unfortunately also in an equally large number of patients scanned for clinically suspected PE, but with a different final diagnosis. The only single parenchymal finding that is

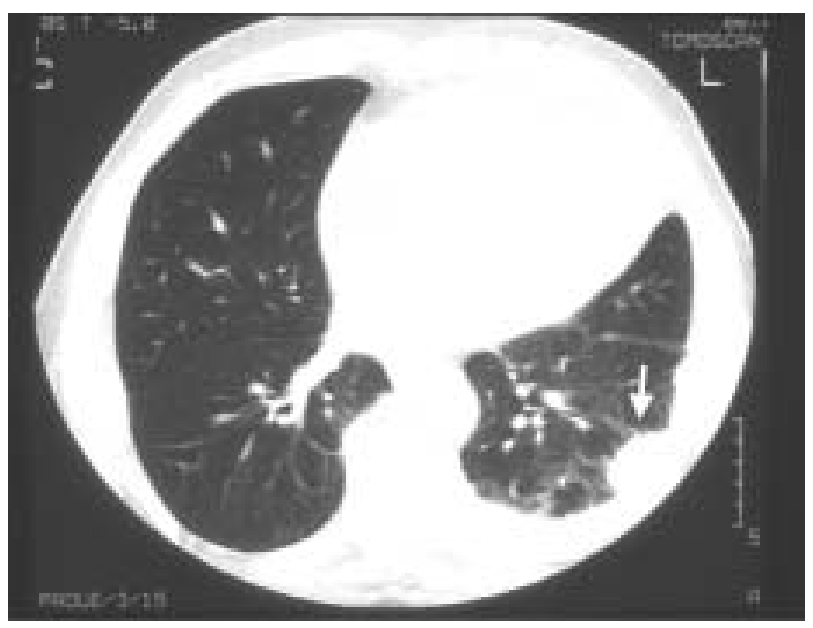

Fig. 2.-Ancillary parenchymal finding in pulmonary embolism (PE). The lung window settings of this computed tomography angiogram in a 54-yr-old male with PE in the left lower lobe shows a pleural based density with a convex margin pointing towards the hilum. This lesion (arrow) represents a peripheral pulmonary infarct and is termed "Hampton's hump". In addition, a small left-sided pleural effusion is present.

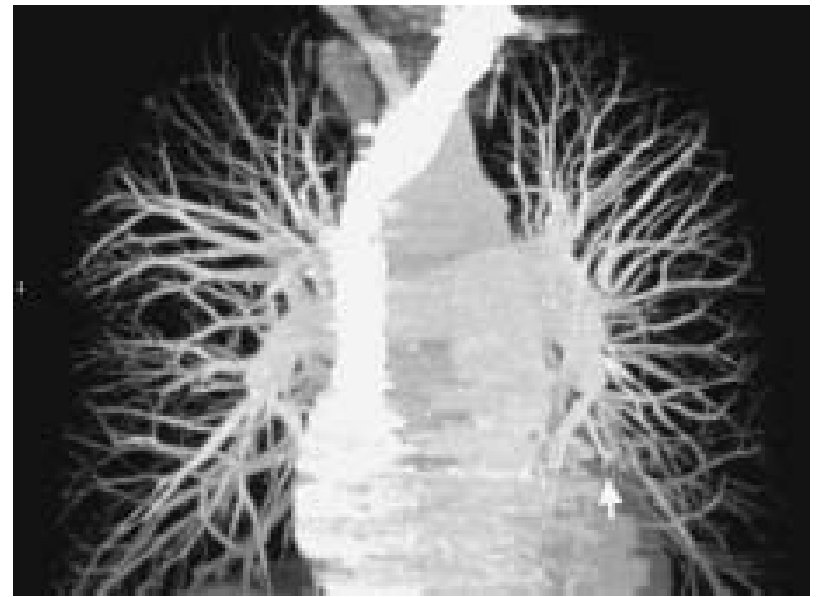

Fig. 3.-Maximum intensity projection image of a multislice computed tomography angiogram of the pulmonary arteries in a patient with suspected pulmonary embolism (PE). Central peripheral pulmonary arteries are shown with excellent detail. PE is present in a left lower lobe segmental artery (arrow) characterized by an abrupt vessel cut-off.

significantly associated with PE is a subpleural wedgeshaped density, commonly known as a Hampton's hump (fig. 2) [16].

Pitfalls in the interpretation of spiral CT arteriograms may be due to insufficient or heterogeneous vascular opacification in patients with cardiovascular and/or pulmonary comorbidity, breathing artifacts, and prominent lymphoid tissue in close vicinity to pulmonary arteries in the hilar area. Occasionally, such perivascular tissue may be confused with intravascular embolic material and thus mimic PE. In unclear cases, the additional use of viewing and image-rendering tools, such as cine-mode analysis, maximum intensity projection and multiplanar and three-dimensional image analysis, may be helpful to avoid misinterpretation (fig. 3) [14, 15]. Another potential pitfall that primarily affects untrained observers is the confusion between acute $\mathrm{PE}$ and chronic thromboembolic disease. Eccentrically located, potentially calcified masses within the pulmonary arteries, contiguous to the vessel wall, abrupt cut-off of lobar or segmental arteries, and irregularities of the vessel diameter are considered findings suggestive of chronic PE (fig. 4) [18].

\section{How to use computed tomography angiography in the evaluation of patients with suspected pulmonary embolism}

Within the last several years, SCTA has been incorporated into the standardized work-up of patients with suspected PE in many institutions. Based on the known advantages of the method and the data documented in the literature, SCTA is most frequently used as the primary imaging method, or as a secondary tool in cases of inconclusive $V / Q$ scintigraphy.

In light of these developments, the advantages and disadvantages of SCTA, the sensitivity and specificity 

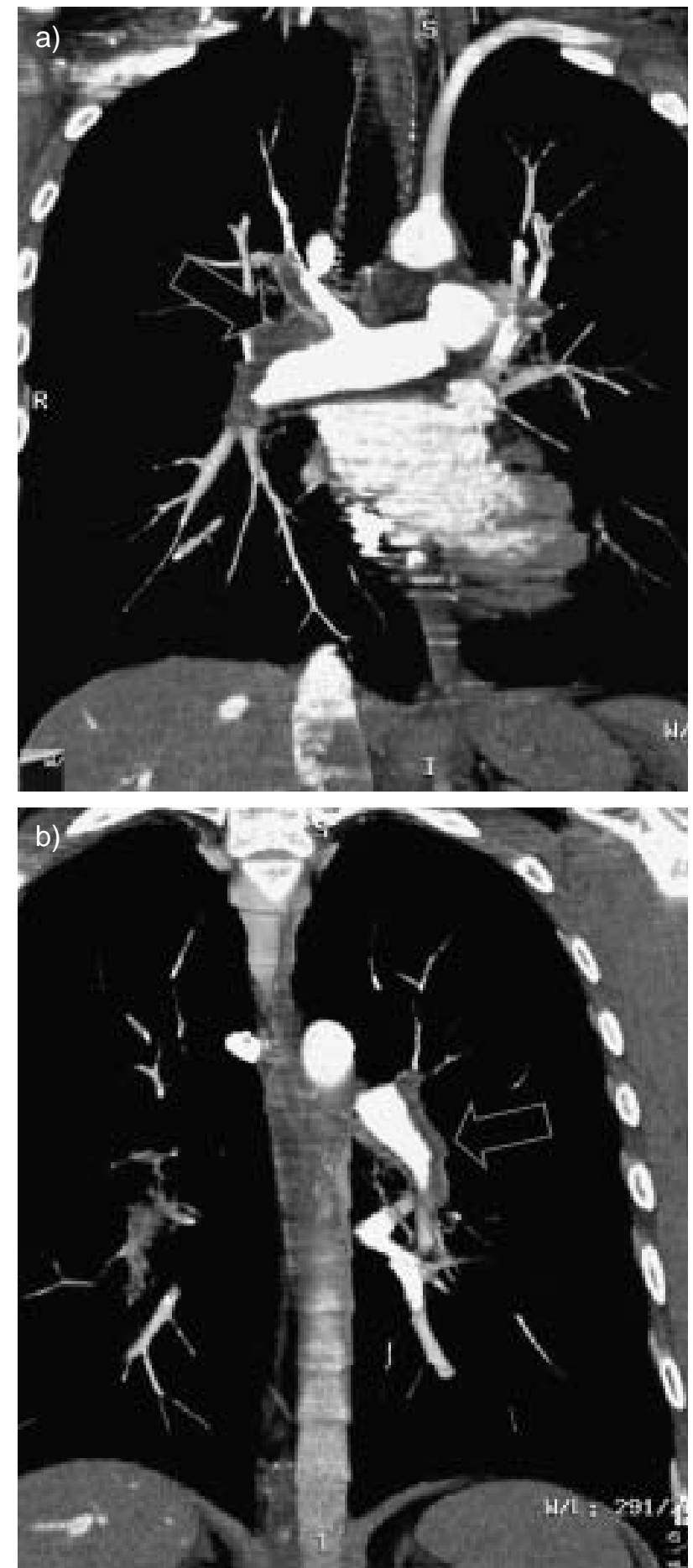

Fig. 4.-Multislice computed tomography angiogram in a patient with chronic thromboembolic pulmonary hypertension. Coronal image reformations demonstrate layers of thrombotic material, excentrically located in the a) right and b) left main pulmonary arteries and extending into the lower lobe vessels (arrows). Reproduced with permission from [17].

of the test, and other criteria, such as cost-effectiveness and outcome research, need to be discussed. In the following, some of these parameters will be revisited to elucidate the basis for current practice in spiral CT imaging in suspected PE.

\section{Accuracy of spiral computed tomography angiography in suspected pulmonary embolism}

Initial studies have reported sensitivities and specificities for single-slice spiral CT in the evaluation of $\mathrm{PE}$ that approach $100 \%$, compared to pulmonary angiography [7, 8]. More recent studies, however, have lowered and broadened the sensitivity and specificity spectrum of SCTA, with sensitivities reported from $53-89 \%$, and specificities from $78-100 \%$ $[9,12,19-22]$. The reasons for such differences in reported accuracies include differences in study design, heterogeneous investigator experience with SCTA, and variations in the anatomical range of the pulmonary vascular tree studied.

In principle, SCTA provides excellent results for the detection of emboli located in the main, lobar, and segmental pulmonary arteries. However, where emboli are located in subsegmental and more peripheral arteries only (fig. 5), the sensitivity of single detector spiral CT seems to be limited $[19,21]$. The substance and significance of this limitation is not totally clear. First, isolated small peripheral emboli are probably rare in clinically symptomatic patients. In two large prospective studies investigating patients with suspected PE, isolated subsegmental emboli were rare and occurred in $4-6 \%$ of patients $[5,23]$. These numbers seem to be more reliable than those from studies with smaller and selected patient samples, which report prevalences of isolated small subsegmental PEs of $\leqslant 30 \%[19,24]$.

Second, the clinical relevance of isolated peripheral subsegmental emboli is not well-defined. Very likely, these small emboli are relatively common even in healthy persons, and do not cause any significant

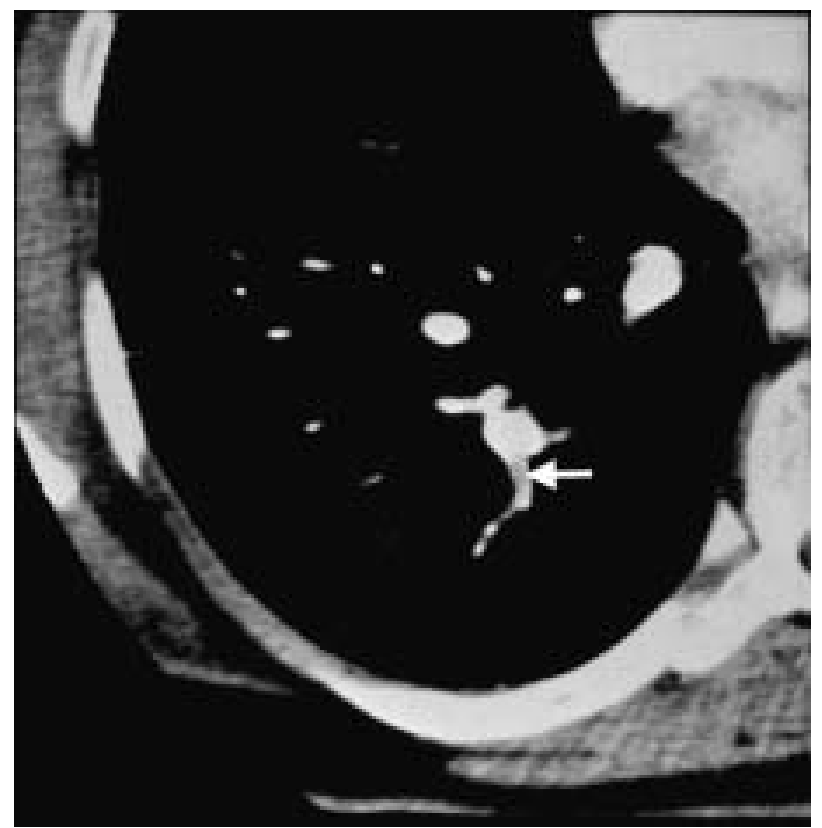

Fig. 5.- Isolated subsegmental embolus. Single slice spiral computed tomography angiogram in a 49-yr-old male shows an isolated small embolus in a subsegmental branch of the posterior basal segment of the right lower lobe (arrow). Note that the other pulmonary arteries in this image demonstrate a normal appearance. 
problem in patients with no other concurrent medical, and particularly, cardiovascular condition [25]. Conversely, in patients with limited cardiopulmonary reserve, even small emboli with a diameter of a few millimeters may cause pulmonary or cardiac problems, or even death. Nevertheless, overlooking a small isolated peripheral clot at SCTA in a patient with suspected PE very likely does not result in an immediate medical problem, since the patient has survived the acute event. In such a patient, however, it is important to investigate the lower extremity veins in order to discover or rule out the presence of thrombotic material that could (re)embolize into the lungs. For this purpose, both ultrasound and contrast venography are suitable. Nevertheless, it is most advantageous to use contrast venography since it is the accepted gold standard, especially in the analysis of the calf veins.

Initial experience with multislice CT indicates that this method will further refine the diagnostic approach to PE, particularly in the diagnosis of small subsegmental emboli (fig. 6). QuANADLI et al. [26] have recently investigated the accuracy of dual-slice spiral CT (a spiral CT scanner with two rows of detectors, and consequently, better performance) for the analysis of suspected PE. These investigators found, in a large cohort of patients, a sensitivity for CT angiography of $90 \%$, and a specificity of $94 \%$ [26]. As noted, it can be expected that the use of scanners with 4,8 , or 16 detector rows will result in a further increase of the accuracy of the method.

Another important consideration in the determination of the accuracy of SCTA is whether pulmonary angiography should be the only gold standard against which spiral CT is measured. A recent report in the literature compared both SCTA and pulmonary angiography against an independent gold standard in a porcine model of the pulmonary arterial tree and found no significant differences in sensitivity or specificity in the diagnosis of PE [27]. In this study, both SCTA with thin collimation and pulmonary angiography had a sensitivity of $87 \%$ in the detection of emboli. Such in vitro studies represent an excellent basis for the independent evaluation of a new modality. However, there are also indications from clinical trials that SCTA may have a similar if not better performance in comparison to pulmonary angiography. In a retrospective analysis of cases with discordant results between SCTA and pulmonary angiography, an expert panel found the initial diagnosis of SCTA to be correct more often than the prospective diagnosis of pulmonary angiography [28]. This notion further erodes the role of pulmonary angiography as the sole and undisputed gold standard in the diagnosis of PE and suggests that the accuracy of SCTA may be higher than that reported to date.

\section{Cost-effectiveness}

SCTA seems to be a cost-effective method, as reported in a study by VAN ERKEL et al. [29], who investigated different combinations of tests to diagnose PE. The authors showed that among 15
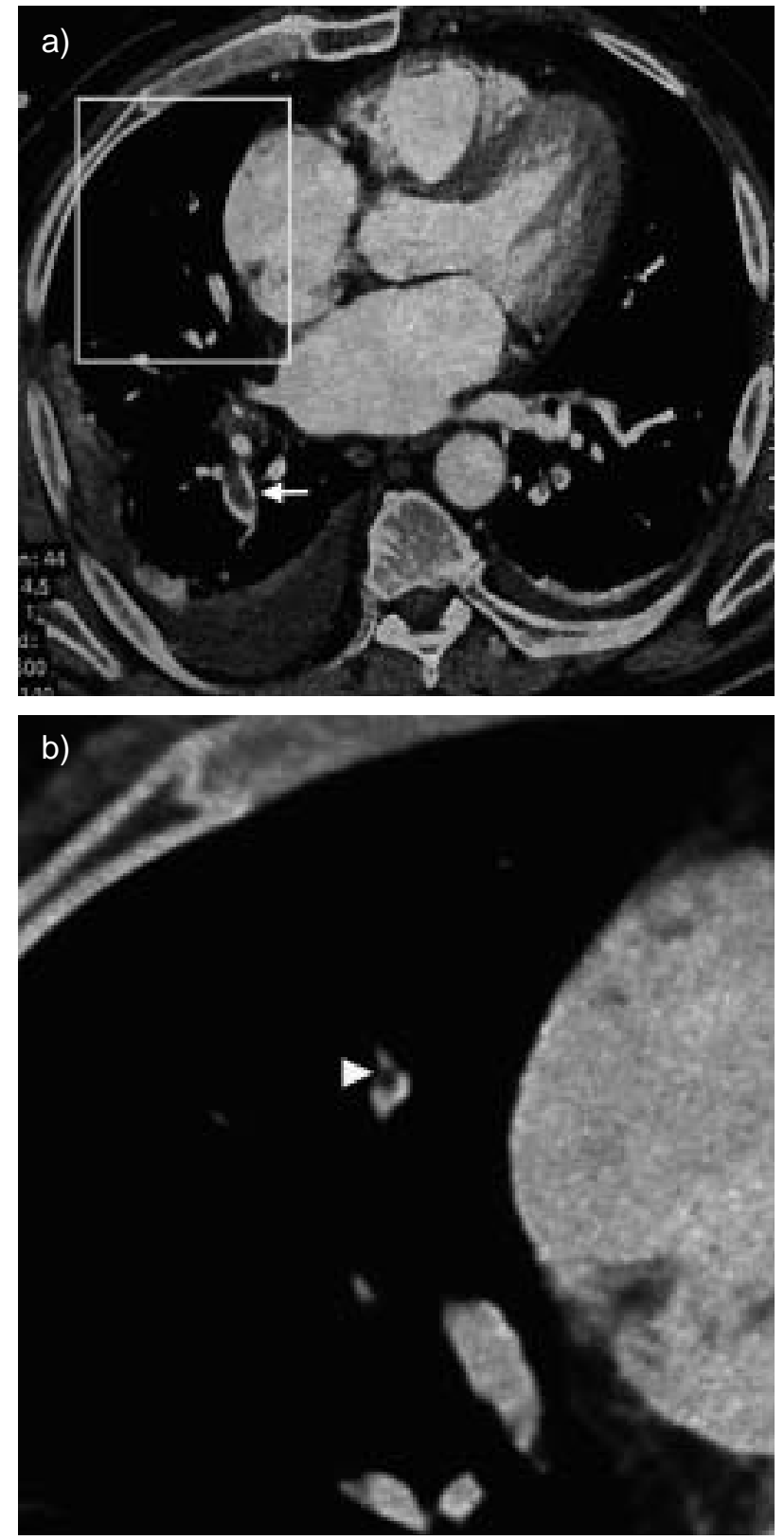

Fig. 6.-Multislice spiral computed tomography (CT) angiogram in pulmonary embolism in a 63-yr-old male patient. a) This multislice CT angiogram, acquired with $2.5-\mathrm{mm}$ slice thickness and a pitch of 6 shows emboli in the right lower lobe artery (arrow) surrounded by contrast enhanced blood. b) Magnified view of inset box, a small subsegmental embolus is also present in the middle lobe (arrow head)

combinations of six diagnostic tests the five most effective strategies (with the lowest cost per life saved and the least mortality) all included SCTA of the pulmonary arteries, in combination with lower limb ultrasonography.

There are a number of caveats that apply to the interpretation of such studies. First, one must understand that in the rather complex cost-effectiveness models, the cost-effectiveness ratios and the strategies recommended depend on numerous variables. If, for example, in the VAN ERKEL et al. [29] model threshold 
sensitivity analysis was performed with mortality as an outcome parameter, and the sensitivity of SCTA was $<85 \%$, the cost-effectiveness ratio and, consequently, the optimal imaging strategy, would change to include $V I Q$ scintigraphy, SCTA, and ultrasound of the leg veins.

The notion that SCTA is a cost-effective test was further supported by a subsequent study by VAN ERKEL et al. [30], who analysed the cost-effectiveness of SCTA in different countries and healthcare systems. As expected, there were considerable differences in the costs for diagnosis and treatment of PE. However, in all countries and systems, the combination of SCTA of the pulmonary arteries, and ultrasound of the leg veins again yielded the best cost-effectiveness ratio, i.e. the lowest costs at the highest survival [30].

\section{Clinical outcome}

Because of the potential limitations of SCTA, and the concerns arising with respect to its sensitivity for demonstrating small clots, clinical outcome studies were undertaken to analyse the clinical value of the method. These studies suggest that clinical outcome is favorable when SCTA does not show PE, and anticoagulation is withheld. For example, FERRETTI et al. [31] followed a group of 164 patients with clinically suspected PE, intermediate probability at $V I Q$ scanning, and a negative result at SCTA. Of the 164 patients with negative SCTA and initially negative results at Duplex ultrasound of the leg, three were found to have clots in the calf veins at short-term follow-up, and were categorized as initially falsenegative on spiral CT angiograms. The authors concluded that in patients with negative spiral CT, clinical outcome was comparable to that in patients with negative $V / Q$ scintigraphy or negative pulmonary angiography. In the author's institution, a retrospective study of patients who underwent SCTA for suspected PE between 1993-1995 showed that in 260 cases with negative SCTA, where anticoagulation was withheld, there was only one recurrent PE $(0.4 \%)$ [32]. Recently, Goodman et al. [33] followed patients who had negative imaging results and who were not anticoagulated for 3 months. Subsequently, PE was found in two (1\%) of 198 patients with negative CT scans (compared to none of the 188 patients with negative $V / Q$ scans). Finally, a yet unpublished study by SWENSEN et al. [34] showed that SCTA was safe and reliable for excluding clinically relevant PE. In $>1,000$ patients with clinically suspected PE and a negative spiral CT scan, where anticoagulation was withheld, the negative predictive value of the negative exam was $99 \%$ [34]. Overall, currently available data suggest that SCTA has a similar negative predictive value compared to pulmonary angiography and $V / Q$ scintigraphy, and that it is safe to withhold anticoagulation in patients with suspected PE and a negative spiral CT angiogram.

\section{Alternative diagnoses}

One of the great advantages of spiral CT over other imaging methods is that spiral CT is not only an angiographic method, but also a standard and routinely used test in the evaluation of other disorders and anatomic structures in the chest. In this context, SCTA provides information not only on the pulmonary arteries, but also on the lung parenchyma, the hila, the mediastinum, and the heart. If it is considered that clinical symptoms in suspected PE are rather nonspecific, and many patients have diagnoses other than PE, it is important to emphasize that SCTA may help to identify alternative findings, and to establish alternative diagnoses (fig. 7) such as aortic dissection in a large number of patients. As KIM et al. [35] demonstrated, SCTA provided additional information and helped to establish alternative diagnoses in $>60 \%$ of patients. The spectrum of alternative diagnoses included pulmonary disorders such as pneumonia or fibrosis, pleural abnormalities, and cardiovascular disease.

\section{One-stop shopping}

The one-stop-shopping philosophy characterizes a diagnostic approach that exploits the possibility of using a single test to answer different questions, and thus, to solve (ideally) a series of problems. In this sense, SCTA is an elegant method to identify, among various clinical differential diagnoses, the one entity potentially responsible for a patient's symptoms, i.e. $\mathrm{PE}$ or, as outlined earlier, an alternative disorder. Clearly, similar information cannot be obtained with $V / Q$ scintigraphy, and is difficult to extract from pulmonary angiography. This notion is supported in a

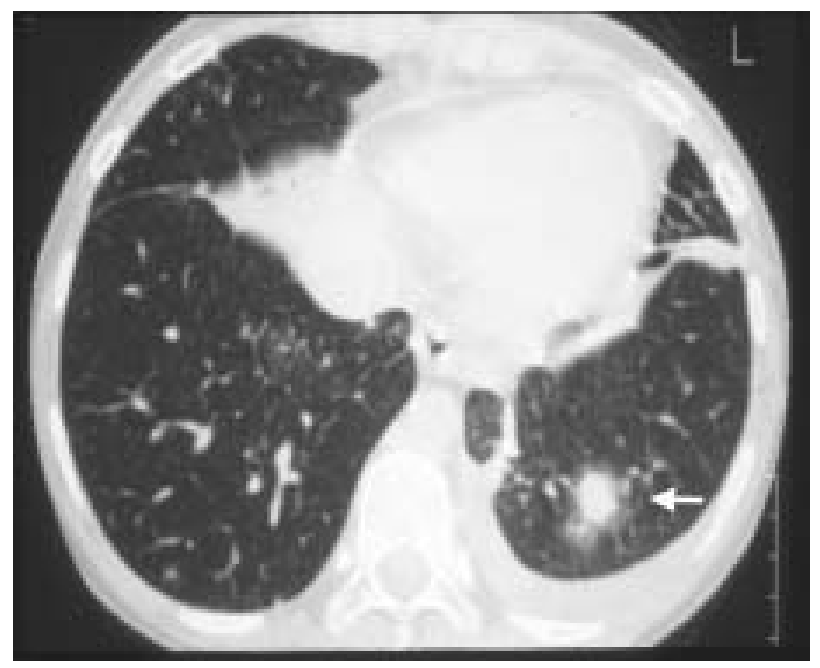

Fig. 7.-Alternative computed tomography (CT) diagnosis in a renal transplant patient with left sided chest pain. A CT angiogram was performed to investigate the presence of pulmonary embolism (PE). Whereas PE was not found, a nodular lesion was detected in the left lower lobe, surrounded by a halo phenomenon (arrow). In addition, a left sided pleural effusion was present. The lesion was biopsied and found to represent post transplant lymphoproliferative disease. 
study by MAYO et al. [20], comparing SCTA and VIQ scintigraphy in a series of patients with suspected PE. In this study, in which pulmonary angiography was used in all cases with inconclusive $V I Q$ scans and in all patients with contradictory results at SCTA and $V I Q$ scanning, SCTA had a sensitivity of $87 \%$, compared to $65 \%$ for $V / Q$ scanning [20]. The specificities of both methods were similar. The authors of this study conclude that their results supported the proposal that SCTA could be used as the initial imaging modality in the diagnosis of PE.

Another approach to one-stop-shopping is the assessment of the pulmonary arterial system and the venous system of the lower extremities in a single imaging session. The pathophysiological background of such a combined assessment is based on the fact that $\mathrm{PE}$ and venous thrombosis are different aspects of the same disorder. Combined SCTA and CT venography exploit the contrast bolus given for the assessment of the pulmonary arteries for the venous phase CT evaluation of the lower extremity veins (fig. 8). As suggested by Loud et al. [36], a CT venogram of the lower extremity veins should be performed 3-4 min after the initial contrast bolus for the lung study has been administered. The pelvic and lower extremity veins are investigated using singlesection scans with $3-5 \mathrm{~mm}$ section thickness every $3-5 \mathrm{~cm}$. In this trial, CT venography had a sensitivity of $97 \%$ and a specificity of $100 \%$ in the detection of venous thrombosis of the lower extremities [36]. The advantages of combining SCTA of the pulmonary arteries and CT venography of the lower extremity veins are clear. First, the diagnosis of venous thromboembolism can be made or excluded within a single session; second, as a consequence, time to diagnosis is shorter; and third, patients with a suspected venous thromboembolism do not have to undergo several time-consuming examinations. It may be argued that the addition of CT venography would substantially increase ionizing radiation; however, if a small number of single thin sections are obtained rather than a full spiral CT examination, the patient is subjected to very little additional ionizing radiation.

\section{Equipment availability}

One issue that is chronically neglected in the discussion of the role of imaging methods in the evaluation of patients with suspected PE is equipment availability. In contrast to the USA, where equipment standards are relatively well-defined for primary, secondary, and tertiary referral centres, the equipment situation in European hospitals is more heterogeneous. This was demonstrated by BuRKILL et al. [37] in a survey regarding equipment availability and imaging strategies used in British hospitals. Pulmonary angiography, one of the two imaging components of the traditional imaging approach to PE, was available in $62 \%$ of hospitals [37]. On the other hand, spiral CT services were provided by $52 \%$ of hospitals, and the combination of $V I Q$ scintigraphy and SCTA were present in only $29 \%$ of hospitals. In another survey, which examined the imaging equipment available for the diagnosis of PE in Austrian hospitals, the present authors group has demonstrated that in 1998, spiral CT was available in $54 \%$, angiography in $46 \%$, and $V I Q$ scintigraphy in $22 \%$ of hospitals, respectively. It is particularly remarkable that in hospitals with $<150$ beds, $V I Q$ scintigraphy was available only in $5 \%$ of institutions [38]. From these results, it can be deduced that imaging in PE cannot rely on a single methodological approach, but rather on the use of the equipment available and on exploiting the different imaging possibilities at hand. Nevertheless, it seems that the availability of SCTA is already exceeding that of $V / Q$ scintigraphy in some European countries or regions.

\section{Limitations of spiral computed tomography angiography}

SCTA has advantages as well as limitations. As outlined earlier, the sensitivity of this method in the detection of isolated small peripheral emboli is one well-known limitation of single-slice spiral CT. This limitation may, however, be overcome by using multislice SCTA with narrow collimation.

Another limitation of SCTA is its susceptibility to breathing artifacts, when a patient is unable to hold their breath during data acquisition. Yet in this case, as well, multislice SCTA with short total scanning times in the range of $10 \mathrm{~s}$ will eventually eliminate the problem. Finally, an inconclusive examination due to insufficient contrast opacification of the pulmonary arteries may represent a problem in patients with heart failure, pulmonary artery hypertension, right-toleft shunts, and a number of other conditions. In the literature, the number of inconclusive exams is reported to range from 1\% [9] to 12\% (European Society of Thoracic Imaging Prospective Investigation of Pulmonary Embolism, Dept of Radiology, University of Vienna, Vienna, Austria). In principle, patients with inconclusive exams at SCTA are also those, who may, due to their comorbidity, have inconclusive results at $V / Q$ scanning or lower quality pulmonary angiograms.

\section{Role of spiral computed tomography in the evaluation of pulmonary embolism}

Because of the disadvantages of the traditional diagnostic methods, and based on the evidence that CT can depict pulmonary emboli with good sensitivity and specificity, it seems reasonable to incorporate this modality into clinical practice [39]. The question is where SCTA should be placed in the numerous diagnostic algorithms which have been designed so far to structure the imaging approach to the diagnosis of PE.

There are several arguments to be made for spiral CT imaging used as the first noninvasive screening procedure for PE, or as a secondary test following inconclusive $V / Q$ scanning. Numerous authors, among them GoODMAN et al. [39], have proposed SCTA as a primary screening procedure for PE and hinted that SCTA could completely replace $V / Q$ scanning. The arguments for using SCTA and 
completely eliminating $V / Q$ scanning include the superior accuracy of spiral $\mathrm{CT}$ in comparison to $V / Q$ scanning as a single method, the superior interobserver agreement of spiral CT [40] and the large number of inconclusive $V / Q$ scans [5]. Nevertheless, there remains a reasonable indication for using $V / Q$ scintigraphy as a primary imaging modality in healthy outpatients without known lung disease.

A discussion on whether spiral CT or pulmonary angiography should be used as a secondary test following inconclusive $V / Q$ scans maybe rather short. Too many publications have reported, and too many institutions have experienced that pulmonary angiography is clinically underused because of its invasiveness and its rather low degree of acceptance by clinicians. Furthermore, as documented by studies on equipment availability $[37,38]$ the access to pulmonary angiography is limited. Therefore, pulmonary angiography will, in the future, be kept to local or regional importance and will otherwise be completly replaced by SCTA.

Considering the current knowledge about the accuracy of different methods in the assessment of $\mathrm{PE}$, and the heterogeneity in method availability and acceptance, it is clear that, for the near future, several different diagnostic strategies and algorithms will coexist. In addition, it seems that spiral CT will play an increasingly important role. At this point, spiral CT has already been incorporated into the clinical routine in many institutions [41]. Spiral CT is already used as a primary screening test for PE where radionuclide lung imaging is unavailable, and in this context, spiral CT can be regarded as sensitive and specific enough to diagnose or rule out relevant PE. Spiral $\mathrm{CT}$, combined with radionuclide lung imaging, is more acceptable to clinicians than a strategy involving pulmonary arteriography, and thus reduces the potentially dangerous "best clinical guess" approach.

Spiral CT is feasible even for critically ill or intubated patients, and spiral CT is now the first imaging method of choice at the author's institution for intensive care patients with suspected PE. In addition, spiral $\mathrm{CT}$ can be useful for monitoring patients who undergo thrombolytic therapy [42, 43]. In these patients, CT allows the visualization of embolic material without the need for a central venous puncture.

\section{Conclusion}

Recent data support the concept that single and multislice spiral computed tomography has fundamentally modified the diagnostic approach to the evaluation of patients with suspected pulmonary embolism. Although the definitive role of spiral computed tomography in the diagnostic algorithm has yet to be determined, it is clear that spiral computed tomography has several advantages compared to established techniques. Patients, as well as physicians, may benefit from this novel approach, which will have to be tailored to the specific needs of individual diagnostic facilities, technical settings, and individual patients.

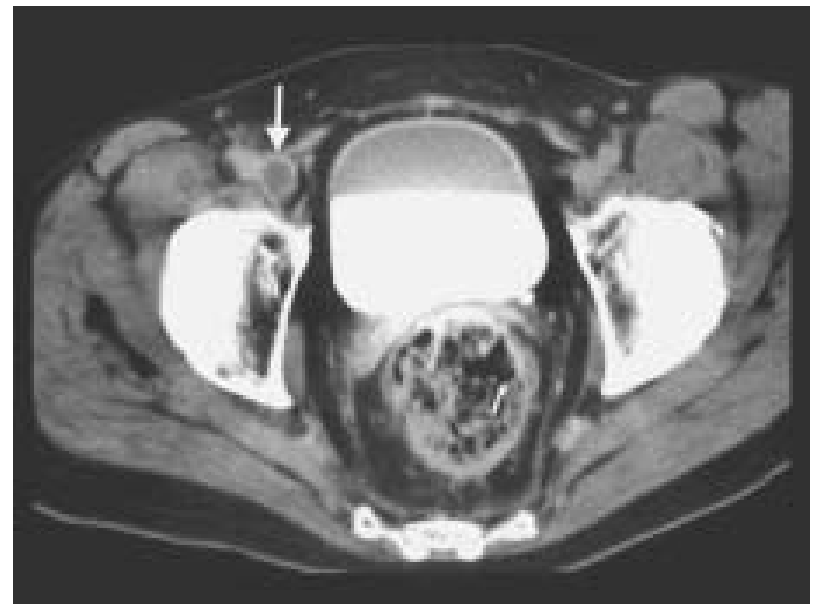

Fig. 8.-Computed tomography (CT) diagnosis of venous thrombosis. A CT venogram, performed in a patient with suspected pulmonary embolism and exploiting the contrast material injected for spiral CT angiography of the pulmonary arteries, shows a large clot in the right femoral vein (arrow). Please note that the contrast of the CT venous phase is sufficient to delineate thrombosis.

\section{References}

1. Dalen JE, Alpert JS. Natural history of pulmonary embolism. Prog Cardiovasc Dis 1975; 17: 257-270.

2. Barritt DW, Jordan SC. Anticoagulant drugs in the treatment of pulmonary embolism: A controlled study. Lancet 1960; 1: 1309-1312.

3. Hull RD, Raskob GE, Caotes G, Panju AA. Clinical validity of a normal perfusion lung scan in patients with suspected pulmonary embolism. Chest 1990; 97: 23-26.

4. Stein PD, Athanasoulis C, Alav A, et al. Complications and validity of pulmonary angiography in acute pulmonary embolism. Circulation 1992; 85: 462-468.

5. PIOPED investigators. Value of the ventilation/perfusion scan in acute pulmonary embolism. Results of the prospective investigation of pulmonary embolism diagnosis (PIOPED). JAMA 1990; 263: 2753-2759.

6. Schluger N, Henschke C, King T, et al. Diagnosis of pulmonary embolism at a large teaching hospital. J Thorac Imaging 1994; 9: 180-184.

7. Remy-Jardin M, Remy J, Wattinne L, Giraud F. Central pulmonary thromboembolism: diagnosis with spiral volumetric CT with single-breath-hold technique-comparison with pulmonary angiography. Radiology 1992; 185: 381-387.

8. Teigen CL, Maus TP, Sheedy II PF, Johnson CM, Stanson AW, Welch TJ. Pulmonary embolism: diagnosis with electron-beam CT. Radiology 1993; 188: 893-845.

9. van Rossum AB, Pattynama PMT, Tjin A, et al. Pulmonary embolism: Validation of spiral CT angiography in 124 patients. Radiology 1996; 201: 467-470.

10. Kalender WA, Polacin A. Physical performance characteristics of spiral CT scanning. Med Phys 1991; 18: 910-915.

11. Remy-Jardin M, Remy J, Artaud D, Deschildre F, Duhamel A. Peripheral pulmonary arteries: Optimization of the spiral CT acquisition protocol. Radiology 1997; 204: 157-163.

12. Remy-Jardin M, Remy J, Deschildre F, et al. Diagnosis of pulmonary embolism with spiral CT: compar- 
ison with pulmonary angiography and scintigraphy. Radiology 1996; 200: 699-706.

13. Schnyder P, Meuli R, Wicky S, Mayor B. Injection techniques in helical CT of the chest. Eur Radiol 1995; 5: 26-33.

14. Herold CJ, Kontrus M, Ziesche G, Fleischmann D, Wegerle T, Huebsch P. Evaluation of pulmonary embolism: value of 3D and multiplanar cine spiral CT angiography. Radiology 1993; 189(P): 264.

15. Remy-Jardin M, Remy J, Cauvain O, Petyt L, Wannebroucq J, Beregi JP. Diagnosis of central pulmonary embolism with helical CT: role of two-dimensional multiplanar reformations. AJR 1992; 165: 1131-1138.

16. Coche EE, Mueller NL, Kim K, Wiggs B, Mazo J. Acute pulmonary embolism: ancillary findings at spiral CT. Radiology 1998; 207: 753-758.

17. Fleischmann D, Scholten C, Klepetko W, Lang IM. Three-dimensional visualization of pulmonary thromboemboli in chronic thromboembolic pulmonary hypertension with multiple detector-row spiral computed tomography. Circulation 2001; 103: 2993.

18. Schwickert HC, Schweden FJ, Schild HH, et al. Pulmonary arteries and lung parenchyma in chronic pulmonary embolism: Preopeative and postoperative CT findings. Radiology 1994; 191: 351-357.

19. Goodman LR, Curtin JJ, Mewissen MW, et al. Detection of pulmonary embolism in patients with unresolved clinical and scintigraphic diagnosis: Helical CT versus angiography. AJR 1995; 164: 1369-1374.

20. Mayo JR, Remy-Jardin M, Muller NL, et al. Pulmonary embolism: prospective comparison of spiral CT with ventilation/perfusion scintigraphy. Radiology 1997; 205: 447-452.

21. Drucker E, Rivity M, Shepard J, et al. Acute pulmonary embolism: assessment of helical CT for diagnosis. Radiology 1998; 209: 235-241.

22. Garg K, Welsh C, Feyerabend A, et al. Pulmonary embolism: diagnosis with spiral CT and ventilation/ perfusion scanning-correlation with pulmonary angiographic results or clinical outcome. Radiology 1998; 208: 201-208.

23. Wallis JW, Kruip M, de Jongh-Leuvenink J, Buller HR. A comparative study of two-rapid D-dimer tests for the exclusion of pulmonary embolism in symptomatic patients. Thromb Haemost 2000; 84: 925.

24. Oser RF, Zuckermann DA, Gutierrez FR, Brink JA. Anatomic distribution of pulmonary emboli at pulmonary angiography: implications for crosssectional imaging. Radiology 1996; 199: 31-35.

25. Gurney JW. No fooling around: Direct visualization of pulmonary embolism. Radiology 1993; 188: 618-619.

26. Quanadli SD, El Hajjam M, Mesurolle B, et al. Pulmonary embolism detection: Prospective evaluation of dual-section helical CT versus selective pulmonary arteriography in 157 patients. Radiology 2000; 217: 447-455.

27. Baile EM, King GG, Muller NL, et al. Spiral computed tomography is comparable to angiography the diagnosis of pulmonary embolism. Am J Respir Crit Care Med 2000; 161: 1010-1015.

28. Herold CJ, Sostman HD, Weber M, Fleischmann D, Hahne J. Determination of retrospective gold standard in patients with suspected PE and discordant results between spiral CT angiography and pulmonary angiography. Radiology 2000; 217: 294.

29. Van Erkel AR, van Rossum AB, Bloem JL, et al. Spiral CT angiography for suspected pulmonary embolism: Cost-effectiveness analysis. Radiology 1996; 201: 29-36.

30. Van Erkel AR, van den Hout WB, Pattynama PM. International differences in health care costs in Europe and United States: Do these affect the costeffectiveness of diagnostic strategies for pulmonary embolism? Eur Radiol 1999; 9: 1926-1931.

31. Ferretti GR, Bosson J-L, Buffaz P-D, et al. Acute pulmonary embolism: Role of helical CT in 164 patients with intermediate probability at $V / Q$ scintigraphy and normal results at duplex US of the legs. Radiology 1997; 205: 453-458.

32. Krestan CR, Klein N, Kreuzer S, Minar E, Janeta J, Herold CJ. Value of a negative spiral-CT angiography in patients with suspected acute PE: Retrospective analysis of PE recurrence and outcome. Eur Radiol 1999; 9: Suppl. 2. 878.

33. Goodman LR, Lipchik RJ, Kuzo RS, Liu Y, McAuliffe TL, O'Brien DJ. Subsequent pulmonary embolism: Risk after a negative helical CT pulmonary angiogram-prospective comparison with scintigraphy. Radiology 2000; 215: 535-542.

34. Swensen SJ, Sheedy PF, Ryu JH, et al. Outcomes after withholding anticoagulation from patients with suspected acute pulmonary embolism and a negative computed tomogram: a cohort study. Mayo Clinic Proceedings 2002 (in press).

35. Kim K, Muller NL, Mayo JR. Clinically suspected pulmonary embolism: Utility of spiral-CT. Radiology 1999; 210: 693-697.

36. Loud PA, Katz DS, Bruce DA, Klippenstein DL, Grossman ZD. Deep venous thrombosis with suspected pulmonary embolism: Detection with combined CT venography and pulmonary angiography. Radiology 2001; 219: 498-502.

37. Burkill GJC, Bell JRG, Padley SPG. Survey on the use of pulmonary scintigraphy, spiral CT and conventional pulmonary angiography for suspected pulmonary embolism in the British isles. Clinical Radiology 1999; 54: 807-810.

38. Schibany N, Fleischmann D, Thallinger C, et al. Equipment availability and diagnostic strategies for suspected pulmonary embolism in Austria. Eur Radiol 2001; 11: 2287-2294.

39. Goodman LR, Lipchik RJ. Diagnosis of acute pulmonary embolism: time for a new approach. Radiology 1996; 199: 25-27.

40. The ESTIPEP investigators. Interobserver agreement in prospective evaluation of pulmonary embolism. Results from the ESTIPEP trial. Eur Radiol 1999; 9: S227.

41. Janata-Schwatczek K, Weiss K, Riezinger I, Bankier A, Domanovits H, Seidler D. Pulmonary embolism II: diagnosis and treatment. Semin Thromb Hemost 1996; 22: $33-52$.

42. Curtin JJ, Mewissen MW, Crain MR, Lipchik RJ. Postcontrast CT in the diagnosis and assessment of response to thrombolysis in massive pulmonary embolism. J Comput Assist Tomogr 1994; 18: 133-135.

43. Remy-Jardin M, Louvegny S, Remy J, et al. Acute central thromboembolic disease: Posttherapeutic followup with spiral CT angiography. Radiology 1997; 203: 173-180.

44. The ESTIPEP investigators. Prospective evaluation of pulmonary embolism: Diagnostic performance of spiral CT angiography in the ESTIPEP trial. Radiology 1999; 213: 126. 